\title{
Adaptive Genetic Fuzzy Decisive Technique Based Moving Target Identification using Multichannel SAR Set-up
}

\author{
Eppili Jaya, B.T. Krishna
}

\begin{abstract}
In the ongoing synthetic aperture radar (SAR) methodology, precise and efficient identification of moving targets is a prominent task. Fractional FT (FrFT)accumulates the energy of the required chirp signal in order to separate it as noise from the chirp.The proposed SAR Moving Target Identification (MTI) process is based on FrFT being combined with the definitive adaptive genetic or neurofuzzy method. The correlation between the transmitted signal and the received signal's FrFT is determined, optimizing the appropriate signal energy and applying it to the decisive adaptive genetic fuzzy unit, which identifies the object location using the fuzzy linguistic rules adaptively. The simulation is conducted by changing the number of targets and number of iterations and the evaluation is performed based on parameters such as missed target rate, detection time and Mean Square Error (MSE), showing that the proposed Adaptive Genetic Fuzzy decisiveMTIsystem located the object with a minimum missed target rate of 0.12 in $5.02 \mathrm{~s}$ and MSE of 23377.4
\end{abstract}

Index Terms: AdaptiveGenetic Fuzzy Decisive technique, multichannel SAR, Fractional FT, function of ambiguity,correlation

\section{INTRODUCTION}

The use in a new generation of areaborne SARs of multiple (competitionary) channels is used for the classical SAR single-channel working in various frequency bands after centuries of achievement. Against a strong ambiguity, the awareness of the moving target provides very important information on both the surveillance and intelligence scenario under observation. FrFT is commonly used in quantum mechanics, photo electrical signal processing and neural artificial networks, signal processing, and so on through the development of the Fractional Fourier transform (FrFT). It has better energy-oriented focusing ability to process non-stationary signals, such as Chirp signal, than conventional Fourier Transform (FT). The FrFT of the chirp signal is more compact than the FT domain so that the sampling frequency of FrFT-related chirps signals can be below the FT level. The FrFT method provides the best refocusing and compute-efficient capabilities for SAR signals. In the SAR method, the Fuzzy Logic concept is capable of managing inconsistencies and ambiguities. The Fuzzy logic system takes care of the data from different remote sensors and generates an easy-to-use gui via adaptive control, avoiding uncertainties for potential remote sensing systems.
The sophisticated SAR information logic approach not only solves the complexity of the SAR signature, it can also provide reliable results by training the data.

The article is structured in the following terms:

The multi-channel SAR MTI method of various approaches is introduced in part I. Section II reveals the computational handling of SAR signals. The adaptive fouzzy genetic approach for moving target identification is proposed in Section III and the results and discussions are presented in Section IV.

\section{MATHEMATICAL MODELLING}

Linear Frequency Modulated (LFM) wave used in Synthetic apertureradar is given as[8],

$$
w_{v}(n, y)=\operatorname{rect}(y \mid p) \cdot \exp \left(j \pi \cdot \chi y^{2}\right) \cdot \exp \left[j 2 \pi f_{c}(n+y)\right](1)
$$

where, $v$-average pulse repetition interval, $n$ - slow time, $p$ width of pulse, $\chi$ - modulation rate, and $f_{c}$-carrier frequency The fast time is denoted as, $y$

The signal of reception is,

$$
u(n, y)=\gamma C \sin c\left[\pi \cdot \beta\left(y-2 K(n) \times \frac{1}{c}\right)\right] \cdot \exp \left[-j 4 \pi K(n) \times \frac{1}{\lambda}\right](2)
$$

where, $\gamma$ is the coefficient of backscattering, $C$ specifies the gains of range compression,

$$
\sin c(a)=\frac{\sin (a)}{a}
$$

The $K(n)$,instantaneous slant range is as,

$$
K(n)=K_{0}-u_{0} \cdot n-b_{0} \cdot \frac{n^{2}}{2}
$$

Substituting equation (4) in equation (2) implies,

$$
\begin{aligned}
& u(n, y)=\gamma C \sin c\left[n \cdot \beta\left(s-2\left(\left[K_{0}-w_{0} \cdot\left[m+\rho\left(m^{\prime}\right)\right) r-b_{0} \cdot \frac{\left[m+\rho\left(m^{\prime}\right)\right) r r^{2}}{2}\right]\right) \times \frac{1}{c}\right)\right] . \\
& \exp \left[-j 4 \pi \frac{K_{0}}{\lambda}\right] \times \exp \left[\left(j 4 \pi \cdot w_{0} \cdot m r+\frac{b_{0}(m r)^{2}}{2}\right) \times \frac{1}{\lambda}\right] \times \\
& \exp \left[j 4 \pi \times\left(u_{0} \rho\left(m^{\prime}\right) \cdot r+b_{0}\left[2 m \rho\left(m^{\prime}\right) r^{2}+\left[\rho\left(m^{\prime}\right) r^{2}\right] \times \frac{1}{2}\right]\right) \times \frac{1}{\lambda}\right]
\end{aligned}
$$

Equation (5) says that the envelope of signal changes because of the target's radial speed and radial acceleration.The moving object is not generally observable with changes in the signal process. 


\section{PROPOSED METHOD OF ADAPTIVE GENETIC FUZZY DECISIVE BASED MOVING TARGET IDENTIFICATION}

The paper's main intention is to track moving objects employing adaptive genetic fuzzy based approach in synthetic aperture radar set-up.. The radar signals change their originality due to the presence of noise, glare, etc., which includes signal processing to accurately detect the position of the moving object within a minimum of time.

The maximum energy of signal is obtained by correlating FrFT signal and original signal. In addition, the function of ambiguity of matched filter signal is correlated with original signal. Thus, two outputs are from matched filter and FrFT are fused using the adaptive geneticfuzzy decision approach. In the fuzzy decision strategy, fuzzy rules are used to expose the moving target in the search area. Fig 1 shows the illustrative diagram of the proposed method of MTI.

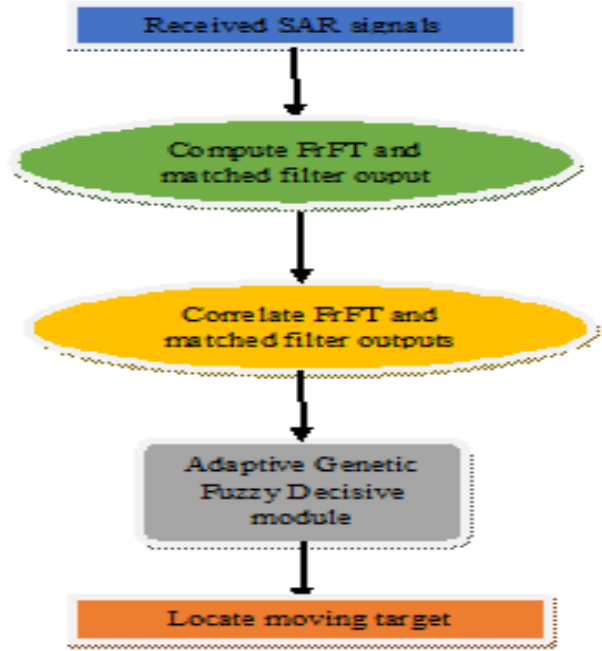

Fig 1.Flowchart execution for the proposed Moving Target Identification (MTI)

A. processing of SAR Signal using FrFT and MF

The uniform samples FrFT is,

$$
\begin{aligned}
& T_{\sigma}[x](g)=\frac{1}{U r} \sum_{m=-\infty}^{\infty} \sum_{n=0}^{N-1} R_{\sigma}\left[g-m \frac{2 \pi \sin \sigma}{U r}-p_{l} \cdot r \cos \sigma\right] \times \\
& e^{j \cdot \frac{1}{2} r^{2} \cdot p_{l}^{2} \cdot \sin \sigma \cos \sigma} \cdot e^{j \frac{2 \pi m}{U r} \cos \sigma\left(g-\frac{\pi m \sin \sigma}{U r}\right)} \cdot e^{-j p_{l} r \cdot \sin \sigma\left(g-\frac{2 \pi m \sin \sigma}{U r}\right)} \cdot e^{-\frac{j 2 \pi n l}{U}}
\end{aligned}
$$

where, $U$ specifies the sampling of non-uniform pointsin each time period $n_{l}$. By applying $\mathrm{AF}$ to the FrFT signal of the original time signal $f(x)$, and is as,

$$
F(0,0)=\int_{-\infty}^{\infty} T_{\sigma}[x](g)^{2} d x
$$

where, $T_{\sigma}[x](g)$ is the FrFT signal of the received signal $f(x), T_{\sigma}^{*}[x](g)$ is the complex conjugate of the FrFT signal with a time shift, and $F(0,0)$ denotes the normalization factor.

- The dispose of correlation is stated as,

$$
\begin{gathered}
\operatorname{Cor}(y, f)=\frac{1}{|\operatorname{Cor}(0,0)|} \int_{-\infty}^{\infty} f(n) \cdot f^{*}(n+y) \cdot e^{j 2 \pi f(n+y)} d n \\
|A(y, f)|=|\operatorname{Cor}(y, f)|
\end{gathered}
$$

where, $\operatorname{Cor}(y, f)$ is the correlation function. The original signal and the time shift in the original received signal are notated as, $f(x)$ and $f^{*}(n+y) e^{j 2 \pi f n} . A(y, f)$ refers to the AF of the original signal, and $|\operatorname{Cor}(y, f)|$ specifies the maximum energy of the original signal.

$$
|F(y, f)|=\left|\operatorname{Cor}^{F}(y, f)\right|
$$

$\mathrm{AF}$ of the original time domain signal and the equation is as,

$$
A(0,0)=\int_{-\infty}^{\infty}|f(n)|^{2} d n
$$

Where, $A(0,0)$ is the factor of normalization, $f(n)$ is the received original signal

For finalizing the position of the target, $T_{\sigma}[x](g)$ and the time time-shift signal $T_{\sigma}^{*}[x](g) \cdot e^{j 2 \pi f(n+y)}$ that gives the peak energy of the signal. $f(n)$ and $T_{\sigma}[u](g)$ are respectively symbolized as, $T_{\text {original }}$ and $T_{F R F T}$.

B. Fusing the decision of FrFTand matched filter adaptively

This section deliberates the fuzzy decisive approach of detecting the moving target in the search area.

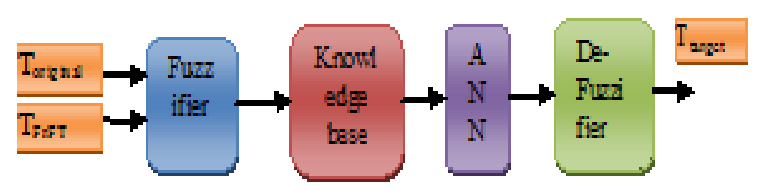

Fig 2. Block diagram of AGFIS model

Fig 2 summates the block diagram of the Adaptive genetic fuzzy inference system (AGFIS), which integrates the prominent features of Fuzzy inference Systems and Artificial Neural Networks (ANN). The two inputs $T_{\text {original }}$ and $T_{F R F T}$ applied to the fuzzification block tomodel the inputs in terms of themembership function of fuzzy which frames using the fuzzy rules to locate the target in the search area. ANN's adaptive capabilities improve system performance.The defuzzifier translates the output variable into the reference crisp values. The required moving target detected using the fuzzy decisive approach is denoted as $T_{t \arg e t}$. The proposed MTI based on AGFIS is capable of reporting rapidly moving targets and being able to effectively and accurately identify multiple targets.

\section{RESULT ANALYSIS AND DISCUSSION}

Figs $3 \& 4$ respectively shows the results of simulation using MATLAB. The objects detected after 50 rounds and 200 rounds are depicted

\section{A. Simulation Results}

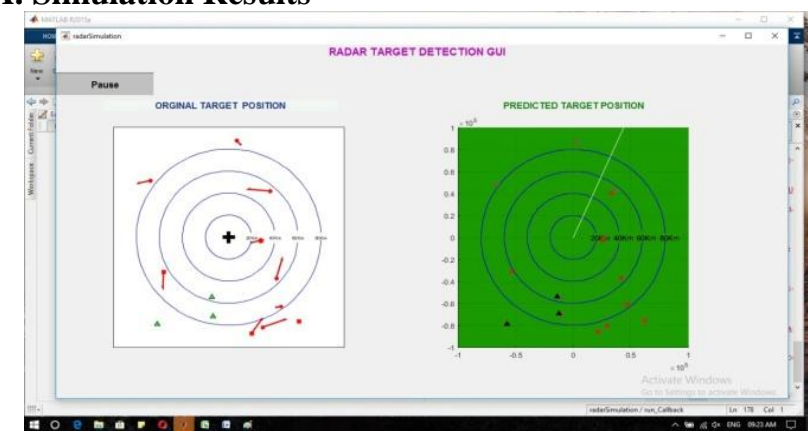

Fig 3. Results of Simulation of moving object identification after 50 rounds

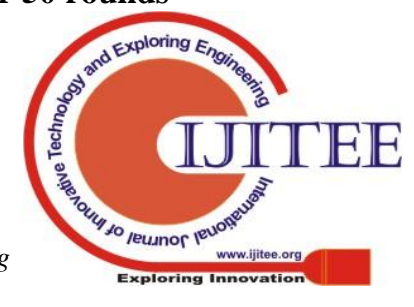
\& Sciences Publication 


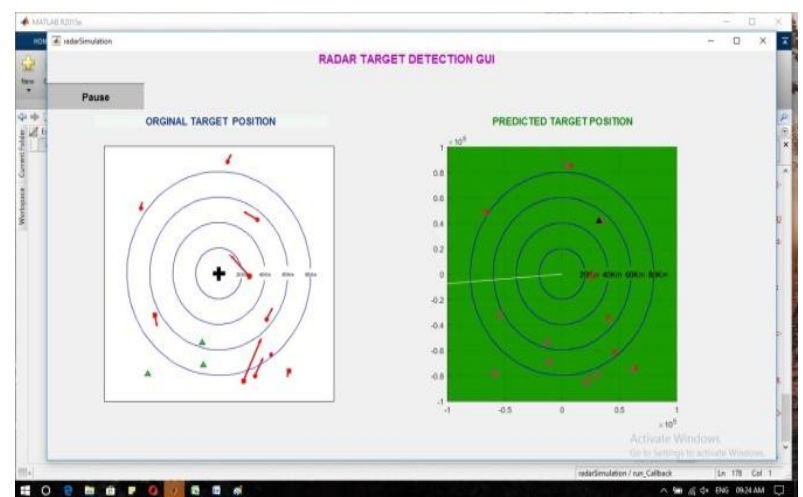

Fig 4. Results of Simulation of moving object identification after 200 rounds

\section{B. Discussion Of Results}

The analysis of number of targets versus missed target rate, identification time and MSE is illustrated in figs 5, 6, 7 respectively by changing $5,10,15$, and 20 targets. It was found that even with more targets, the proposed method provides less time to identify targets, less target rate missed and less MSE compared to existing methods.

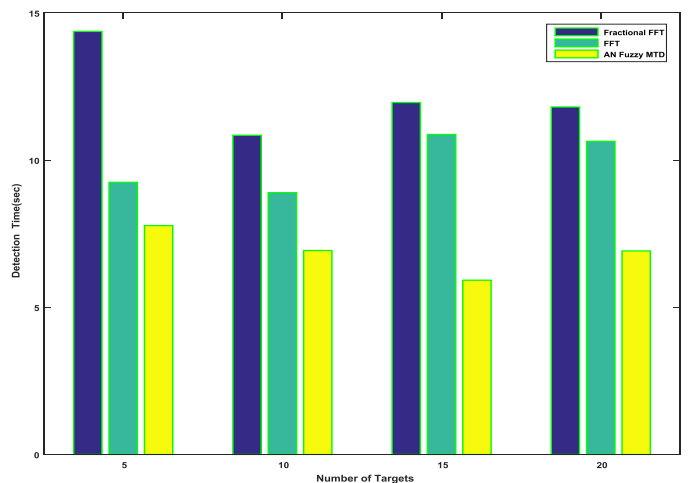

Fig 5. Analysis based on the number of targets versus identification time

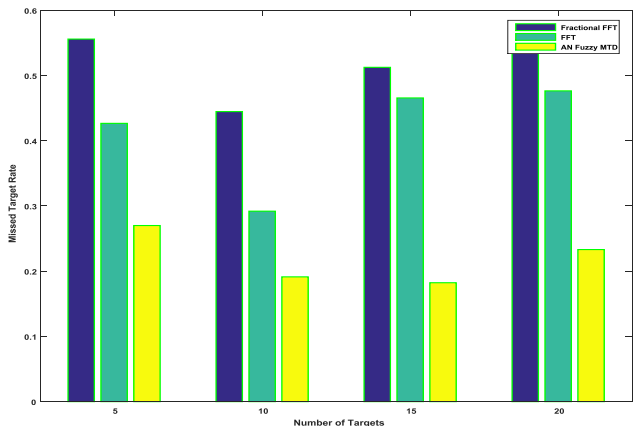

Fig 6. Analysis based on the number of targets versus missed target rate.

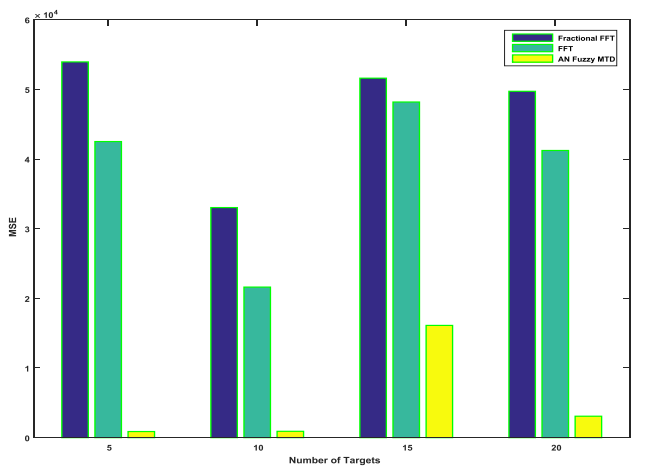

Fig 7. Analysis based on the number of targets versus MSE

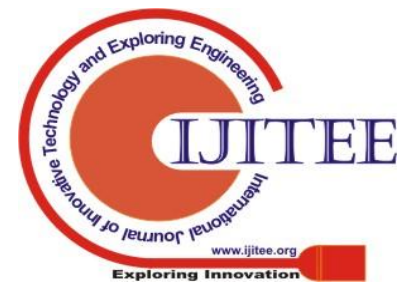


Table I. Analysis of comparison for N=20 targets of the MTI methods

\begin{tabular}{|c|c|c|c|}
\hline & FrFT & FFT & $\begin{array}{c}\text { Proposed } \\
\text { method }\end{array}$ \\
\hline DT (s) & 11.80 & 10.63 & $\mathbf{6 . 9 1}$ \\
\hline MTR & 0.573 & 0.476 & $\mathbf{0 . 2 3 3}$ \\
\hline MSE & 49721.6 & 41225.0 & $\mathbf{3 0 7 7 . 5}$ \\
\hline
\end{tabular}

Table II.Analysis of comparison for $\mathbf{5 0 0 0}$ iterations.of the MTI methods

\begin{tabular}{|c|c|c|c|}
\hline & FrFT & FFT & $\begin{array}{c}\text { Proposed } \\
\text { method }\end{array}$ \\
\hline DT (s) & 12.91 & 11.37 & $\mathbf{6 . 8 8}$ \\
\hline MTR & 0.685 & 0.617 & $\mathbf{0 . 2 6 0}$ \\
\hline MSE & 42043.7 & 41047.7 & $\mathbf{2 9 7 2 . 8}$ \\
\hline
\end{tabular}

\section{CONCLUSION}

The proposed method used the Adaptive genetic Fuzzy based MTIsystem for Synthetic aperture radar signals to detect moving objects in the search area. The proposed method potency is intensified by employing AGFIS model, where fuzzy rules are used adaptively to get accurate output. Up to 20 moving targets andby changing number of iterations, the MATLAB simulation is performed. The execution parameters to measure the efficacy of proposed and current methods are missed target rate, Identification time and MSE. Comparative analysis substantiates that the proposed Adaptive genetic Fuzzy MTIdetect the targetsprecisely with minimum amount of time.

\section{REFERENCES}

1. LudwigMichael, et al., "multi-channel synthetic aperture radar in europe" IGARSS 2017.

2. Z. Li, et al., "Multi-frame fractional Fourier transform technique for moving target detection with space-based passive radar", IET, Radar, Sonar \& Navigation, volume. 11, no. 5, pp. 822-828, May 2017.

3. Yang, J., Liu, C and Wang, Y.: 'Detection and imaging of ground moving targets with real SAR data', IEEE Transactions on Geoscience and Remote Sensing, 2015, 53, no. 2, pp. 920-932.

4. J.R Fienup, "Detecting moving targets in SAR imagery by focusing", IEEE Transactions on Aerospace and Electronic Systems, 2001, 37, (3), pp. 794-809.

5. A Saucan,et al.,"3-D bathymetric reconstruction in multi-path and reverberant underwater environments", in proceedings of 2014 IEEE International Conference on Image Processing (ICIP), 2014, pp. 5831-5835.

\section{AUTHORS PROFILE}

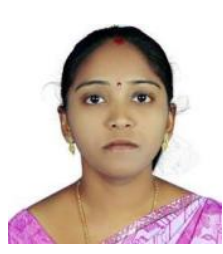

Smt. Eppili Jaya completed her B.Tech and M. Tech from Aditya Institute of Technology and Management, Tekkali in 2009 \& 2011 respectively. Now she is Pursuing Ph.D at JNTU Kakinada, Andhra Pradesh, INDIA and working as Assistant professor in Aditya Institute of Technology and Management, Tekkali, Andhra Pradesh, INDIA. She has ten years of working experience in teachingandactively published sevenresearch papers in various international journals. She also attended various Faculty Development Programs to enhance the knowledge in research areas. Her research interests are Digital Signal processing, VLSI and Internet of Things.

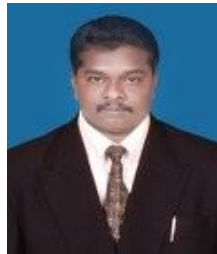

Dr. B. Tirumala Krishna

completed his B.E., M.E., and Ph.D. degrees at the College of Engineering, Andhra University, Visakhapatnam, India. He worked at GITAM University, Visakhapatnam, India, at various levels as Assistant Professor and Senior Assistant Professor. He is currently working as Professor at the Department of ECE, Jawaharlal Nehru Technological University Kakinada (JNTUK), Kakinada, India. His research areas of interest are fractional-order systems, fractional-order signal processing, and analog VLSI. His Bio-Data is placed in Marquis who's who in the world in 2014 2015, 2016 editions. He is a member of IEEE, Fellow of Institute of Electronics and Telecommunication Engineers (FIETE), Life Member Instrument Society of India, Life Member Bio-Medical Engineering Society of India. One of his papers is listed in best downloaded, Best cited articles from Elsevier signal processing journal during 2011, 2012. He has visited Srilanka, Indonesia, Malaysia and Thailand to present paper, chairing technical sessions in various conferences. He has reviewed papers in many international journals of IEEE (USA), Elsevier Journals, Springer Journals and some Conferences. He is currently handling two sponsored research projects each from Department of Science and Technology (DST), New Delhi and University Grants Commission, New Delhi, India 